\title{
COMBINED ECONOMIC AND EMISSION DISPATCH WITH AND WITHOUT CONSIDERING TRANSMISSION LOSS
}

\author{
Pratyush Das ${ }^{1}$, Raju Patwary ${ }^{2}$ and S.C. Konar $^{3}$ \\ ${ }^{1}$ Department of Electrical Engineering, Global Institute of Management and \\ Technology, Krishnagar, Nadia \\ pratyush. 85egmail.com \\ ${ }^{2}$ Department of Electrical Engineering, Gargi Memorial Institute of \\ Technology, Balarampur, Baruipur,24Parganas (S) \\ rajpat_2008@yahoo.com \\ ${ }^{3}$ Department of Electrical Engineering, Bengal Engineering and Science \\ University, Shibpur, Howrah \\ sckonar55@gmail.com
}

\begin{abstract}
This paper gives a complete idea about the Combined Economic and Emission Dispatch (CEED) in different load demand. This paper shows CEED of a six generator system by neglecting the transmission loss first, and after that CEED of the same system considering transmission loss. Here we solve the CEED problem with the help of Mat-Lab software. The results are graphically represented here, like generation cost $\mathrm{v} / \mathrm{s}$ load demands; load shared by each generator in different load demand and transmission loss $v / s$ load demands.
\end{abstract}

\section{KEYWORDS}

Economic Load Dispatch (ELD), Economic Emission Dispatch (EED), Combined Economic and Emission Dispatch (CEED), B-coefficients.

\section{INTRODUCTION}

Economic Load Dispatch means finding out the optimal outputs of the generators to meet the total load demand of a system. There are several different algorithms to solve an ELD problem for different loads with or without considering transmission loss [2], [3], [4]. Generally with the help of ELD, we are benefitted financially. But now a day's throughout the world everyone concentrate on reducing the pollutions. At the time of generation many pollutants like carbon dioxide $\left(\mathrm{CO}_{2}\right)$, oxides of nitrogen $(\mathrm{NOx})$, and oxides of sulphur ( $\left.\mathrm{SO} x\right)$ are produced from the fossil fuels. These pollutants have, mainly, ill effects to the human body. So, for the environmental point of view the Economic Emission Dispatch (EDD) is introduced, to minimize the emissions for a certain load demand. That means at the time of economic operation, we have to take care of emission constraints along with the financial conditions. For this reason by combining EED and ELD, Combined Economic and Emission Dispatch is introduced (CEED).

Different algorithms are used for solving CEED problems [5], [6], [7]. Here a CEED problem for six generator system without considering transmission loss is performed. Then with the help of B-

Rupak Bhattacharyya et al. (Eds) : ACER 2013,

pp. 347-355, 2013. (C) CS \& IT-CSCP 2013

DOI : $10.5121 /$ csit.2013.3232 
coefficient matrix, the CEED problem is performed with consideration of transmission loss. As a result, technical as well as environmental issues can be taken care of.

In this article, in section two, the problem formulation is discussed. In subsection 2.1, Economic Load Dispatch (ELD) is discussed. In subsection 2.2, Economic Emission Dispatch (EED) is discussed. Subsection 2.3 discusses the Combined Economic and Emission Dispatch (CEED). Section 3 includes the algorithms for CEED with and without considering loss. Section 4 contains a numerical example with discussion on the results. Finally, some concluding remarks are specified in section 5 .

\section{Problem Formulation}

The total procedure to solve a CEED problem for a given example is discuss in the following sections.

\subsection{Economic Load Dispatch (ELD)}

Cost function in an Economic Load Dispatch is given by-

$$
\begin{aligned}
& F_{c}=\sum_{i=1}^{N G}\left(A_{i} P g_{i}^{2}+B_{i} P g_{i}+C_{i}\right) R s / h r \\
& \text { Where } \\
& F_{c}=\text { total fuel cost }(\mathrm{Rs} / \mathrm{hr}) \\
& N G=\text { total number of generators } \\
& A_{i}, B_{i} \& C_{i}=\text { cost coefficients of } \mathrm{i}^{\text {th }} \text { generator. } \\
& P g_{i}=\text { output of } \mathrm{i}^{\text {th }} \text { generator. }
\end{aligned}
$$

There are some constraints, which we have to follow. They are-

\subsubsection{Power balance constraint:}

$$
P_{D}+P_{L}=P g_{i}
$$

Where,

$$
\begin{aligned}
& P_{D}=\text { load demand } \\
& P_{L}=\text { transmission loss }
\end{aligned}
$$

Again this $P_{L}$ can be calculated by following formula

$$
P_{L}=\sum_{i=1}^{N G} \sum_{j=1}^{N G} P g_{i} B_{i j} P g_{j} M W
$$

$B_{i j}$ is known as loss coefficient or B-coefficient of B-coefficient matrix. This B-coefficient matrix is very essential for any economic operation considering loss.

\subsubsection{Generating Capacity Limits:}

$$
P g_{i}^{\min } \leq P g_{i} \leq P g_{i}{ }^{\max }
$$

Where, 


$$
\begin{aligned}
& P g_{i_{\min }}=\text { Minimum power output limit of } \mathrm{i}^{\text {th }} \text { generator } \\
& P g_{i_{\max }}=\text { Maximum power output limit of } \mathrm{i}^{\text {th }} \text { generator }
\end{aligned}
$$

\subsection{Economic Emission Dispatch (EED)}

In Economic Emission Dispatch, total emission is given by-

$$
\begin{aligned}
E_{C}=\sum_{i=1}^{N G}\left(D_{i} P g_{i} 2+E_{i} P g_{i}+F_{i}\right) \\
\text { Where, } \\
E_{c}=\text { total emission } \\
D_{i}, E_{i} \& F_{i}=\text { emission coefficients of } i^{\text {th }} \text { unit }
\end{aligned}
$$

\subsection{Combined Economic and Emission Dispatch (CEED)}

These ELD and EED are different to each other. The ELD reduces the fuel cost by increasing the pollutants. Whereas the EED reduces the emission of pollutant gasses by increasing the fuel costs. So, we have to find out a operating point to make a balance between operating cost and emission rate and this can be achieved by CEED.

The main objective function in CEED can be developed by combining ELD with EED with the help of price penalty factor $h_{i}$ (Venkatesh et al.,2003) as follows:

$$
F_{T}=\sum_{i=1}^{N G}\left(\left(A_{i} P g_{i}^{2}+B_{i} P g_{i}+C_{i}\right)+h_{i}\left(D_{i} P g_{i}^{2}+E_{i} P g_{i}+F_{i}\right)\right)
$$

The price penalty factor can be calculated by the following formula:

$$
h_{i}=\frac{A_{i} P g_{i}^{2}+B_{i} P g_{i}+C_{i}}{D_{i} P g_{i}{ }^{2}+E i P g_{i}+F_{i}}
$$

\section{Algorithms}

\subsection{Algorithm for CEED (without considering loss)}

1. Read data, namely cost coefficients, $A_{i}, B_{i}, C_{i} ; D_{i}, E_{i}, F_{\mathrm{i}}$, B- coefficients, $B_{i j}, B_{i 0}, B_{00}(i=1$, $2, \ldots \ldots, N G$ ); convergence tolerance, $\xi$; step size $\alpha$; and maximum iteration allowed, ITMAX, $P g_{i}^{\text {min }}, P g_{i}^{\text {max }}$ etc.

2. Find out hi by equation ( $v i$ ) and find out modified cost coefficients $a_{i}, b_{i} \& c_{i}$

3. The problem can be stated by formulae

$$
\begin{aligned}
& F\left(P g_{i}\right)=\sum_{i=1}^{N G} F_{i}\left(P g_{i}\right) \\
& \text { and } \\
& \sum_{i=1}^{N G} P g_{i}=P_{D}
\end{aligned}
$$


The values of $\lambda$ and $P g_{i}(\mathrm{i}=1,2, \ldots \ldots, \mathrm{NG})$ can be obtained directly using the formulae

$$
\begin{aligned}
& \lambda=\frac{\left(P_{D}+\sum_{i=1}^{N G} \frac{b_{i}}{2 * a_{i}}\right)}{\sum_{i=1}^{N G} \frac{1}{2 * a_{i}}} \\
& \& g_{i}=\frac{\lambda-b_{i}}{2 * c_{i}}
\end{aligned}
$$

4. Assume no generator has been fixed at either lower limit or at upper limit

5. Check the limits of generators, if no more violations then go to step 13 , else fix as following :

6. Go to step 3

$$
\begin{aligned}
& \text { If } P g_{i}<P g_{i}^{\min } \text { then } P g_{i}=P g_{i}^{\text {min }} \\
& \text { If } P g_{i}=P g_{i}^{\text {max }} \text { then } P g_{i}=P g_{i}^{\text {max }}
\end{aligned}
$$

7. Compute optimal total cost from Eq.(v) .

8. STOP

\subsection{Algorithm for CEED (considering loss)}

1. Read data, namely cost coefficients, $A_{i}, B_{i}, C_{i} ; D_{i}, E_{i}, F_{i}$, B- coefficients, $B_{i j}, B_{i 0}, B_{00}(i=1$, $2, \ldots \ldots, N G$ ); convergence tolerance, $\xi$; step size $\alpha$; and maximum iteration allowed, ITMAX, $P g_{i}^{\text {min }}, P g_{i}^{\text {max }}$ etc.

2. Find out $h_{i}$ by equation (vi) and find out modified cost coefficients $a_{i}, b_{i} \& c_{i}$

3. Compute the initial values of $P g_{i}(i=1,2, \ldots \ldots, \mathrm{NG})$ and $\lambda$ by assuming that the transmission losses are zero, i.e. $P_{L}=0$. Then the problem can be stated by formulae

$$
\begin{gathered}
F\left(P g_{i}\right)=\sum_{i=1}^{N G} F_{i}\left(P g_{i}\right) \\
\& \\
\sum_{i=1}^{N G} P g_{i}=P_{D}
\end{gathered}
$$

The values of $\lambda$ and $P g_{i}(i=1,2, \ldots \ldots, N G)$ can be obtained directly using the formulae

$$
\begin{gathered}
\lambda=\frac{\left(P_{D}+\sum_{i=1}^{N G} \frac{b_{i}}{2 * a_{i}}\right)}{\sum_{i=1}^{N G} \frac{1}{2 * a_{i}}} \\
\& \\
P g_{i}=\frac{\lambda-b_{i}}{2 * c_{i}}
\end{gathered}
$$

4. Assume no generator has been fixed at either lower limit or at upper limit.

5. Set iteration counter, IT $=1$.

6. Compute $P g_{i}(i=1,2, \ldots \ldots, \mathrm{R})$ of generators which are not fixed at either upper or lower limits, using the following equation 


$$
P g_{i}=\frac{\lambda\left(1-B_{i 0}-\sum_{\substack{j=1 \\ j \neq i}}^{N G} 2 * B_{i j} * P g_{j}\right)-B_{i}}{2\left(a_{i}+\lambda B_{i i}\right)}
$$

7. Compute transmission loss using Eq.(iii)

8. Compute $\Delta P=P_{D}+P_{L}-\sum_{i=1}^{N G} P g_{i}$

9. Check $\Delta P \leq \xi$, if 'yes then go to step 12

Check IT $\geq$ ITMAX, if 'yes then go to step 11. (it means program terminated without obtaining the required convergence.)

10. Update $\lambda^{\text {new }}=\lambda+\alpha|\Delta P|$, where $\alpha$ is the step size used to increase or decrease the value of $\lambda$ in order to meet the step 8 .

11. IT $=\mathrm{IT}+1, \lambda=\lambda^{\text {new }}$ and go to step 6 and repeat

12. Check the limits of generators, if no more violations then go to step 14, else fix as following :

13. Go to step 5

$$
\begin{aligned}
& \text { If } \quad P g_{i}<P g_{i}^{\min } \text { then } P g_{i}=P g_{i}^{\text {min }} \\
& \text { If } P g_{i}=P g_{i}^{\max } \text { then } P g_{i}=P g_{i}^{\text {max }}
\end{aligned}
$$

14. Compute optimal total cost from Eq.(v) and transmission loss from Eq. (iii).

15. STOP

\section{EXEMPLIFICATION AND DISCUSSION}

\subsection{Inputs}

We are taking here a six generator system. The required generators data for the CEED problem is given in the Table 1. To perform the same problem B-coefficient matrix is also required and it is

\begin{tabular}{|c|c|c|c|c|c|c|c|c|}
\hline Generator & $\begin{array}{c}A_{i}(R s / \\
\left.M W^{2} h r\right)\end{array}$ & $\begin{array}{l}B_{i}(R s / \\
M W h r)\end{array}$ & $\begin{array}{c}C_{i}(R s / \\
h r)\end{array}$ & $\begin{array}{c}D_{i}(\mathrm{Kg} / \\
M W 2 \mathrm{hr})\end{array}$ & $\begin{array}{l}E_{i}(\mathrm{Kg} / \\
M W h r)\end{array}$ & $\begin{array}{c}F_{i}(\mathbf{K g} / \\
h r)\end{array}$ & $\begin{array}{l}P g i_{\max } \\
(M W)\end{array}$ & $\begin{array}{l}P g i_{\text {min }} \\
(M W)\end{array}$ \\
\hline 1 & 0.00375 & 2 & 0 & 0.0126 & -1.1 & 22.983 & 300 & 5 \\
\hline 2 & 0.01750 & 1.75 & 0 & 0.0200 & -0.1 & 25.313 & 80 & 4 \\
\hline 3 & 0.06250 & 1 & 0 & 0.0270 & -0.01 & 25.505 & 85 & 8 \\
\hline 4 & 0.00834 & 3.25 & 0 & 0.0291 & -0.005 & 24.9 & 75 & 3 \\
\hline 5 & 0.02500 & 3 & 0 & 0.0290 & -0.004 & 24.7 & 90 & 5 \\
\hline 6 & 0.02500 & 3 & 0 & 0.0271 & -0.0055 & 25.3 & 90 & 5 \\
\hline
\end{tabular}
given in the Table 2.

Table 1. Generators data

Table 2. B-coefficient matrix for six generator system

\begin{tabular}{|c|c|c|c|c|c|}
\hline 0.0003 & 0.0001 & 0 & -0.0001 & 0 & -0.0001 \\
\hline 0.0001 & 0.0001 & 0.0001 & -0.0001 & 0 & -0.0001 \\
\hline 0 & 0.0001 & 0.0006 & -0.0001 & 0 & -0.0002 \\
\hline-0.0001 & -0.0001 & -0.0001 & 0.0023 & 0.0006 & -0.0002 \\
\hline 0 & 0 & 0 & 0.0006 & 0.0007 & -0.0002 \\
\hline-0.0001 & -0.0001 & -0.0002 & -0.0002 & -0.0002 & 0.0006 \\
\hline
\end{tabular}




\subsection{Figures and Tables of Outputs (without Loss)}

In this section, with the help of the input data (Table 1), we find out the following results (Table 3.) using Mat-Lab software [10]. Here we increase the load demand from $100 \mathrm{MW}$ to $400 \mathrm{MW}$ with an interval of $50 \mathrm{MW}$ and find out the load sharing between the six generators for the corresponding load demands. Sharing of the loads between the all generators for different load is shown in the Table 3.

Table 3.Generator outputs \& costs in different loads

\begin{tabular}{|c|c|c|c|c|c|c|c|}
\hline $\begin{array}{c}\mathbf{L o a d} \\
(\mathbf{M W})\end{array}$ & $\boldsymbol{P g}_{\mathbf{1}}(\mathbf{M W})$ & $\begin{array}{c}\boldsymbol{P g}_{2} \\
(\mathbf{M W})\end{array}$ & $\begin{array}{c}\boldsymbol{P g}_{3} \\
(\mathbf{M W})\end{array}$ & $\begin{array}{c}\boldsymbol{P g}_{4} \\
(\mathbf{M W})\end{array}$ & $\begin{array}{c}\boldsymbol{P g}_{5} \\
(\mathbf{M W})\end{array}$ & $\begin{array}{c}\boldsymbol{P g}_{\boldsymbol{6}} \\
(\mathbf{M W})\end{array}$ & $\begin{array}{c}\text { Cost } \\
(\mathbf{R s} / \boldsymbol{h})\end{array}$ \\
\hline 100 & 52.3783 & 20.6419 & 11.8654 & 4.8586 & 5.1066 & 5.1493 & 227.4 \\
\hline 150 & 80.5859 & 27.4091 & 14.5679 & 9.7275 & 8.8099 & 8.8998 & 363.9 \\
\hline 200 & 108.7935 & 34.1763 & 17.2704 & 14.5964 & 12.5132 & 12.6503 & 510.8 \\
\hline 250 & 137.0010 & 40.9435 & 19.9730 & 19.4652 & 16.2165 & 16.4008 & 667.9 \\
\hline 300 & 165.2086 & 47.7107 & 22.6755 & 24.3341 & 19.9198 & 20.1513 & 835.2 \\
\hline 350 & 193.4162 & 54.4779 & 25.3780 & 29.2030 & 23.6231 & 23.9018 & 1012.9 \\
\hline 400 & 221.6238 & 61.2451 & 28.0805 & 34.0718 & 27.3264 & 27.6524 & 1200.8 \\
\hline
\end{tabular}

Figure 1. shows total generation costs for different load demands graphically and the load shared by the each generator for different loads is graphically shown in the Figure 2.

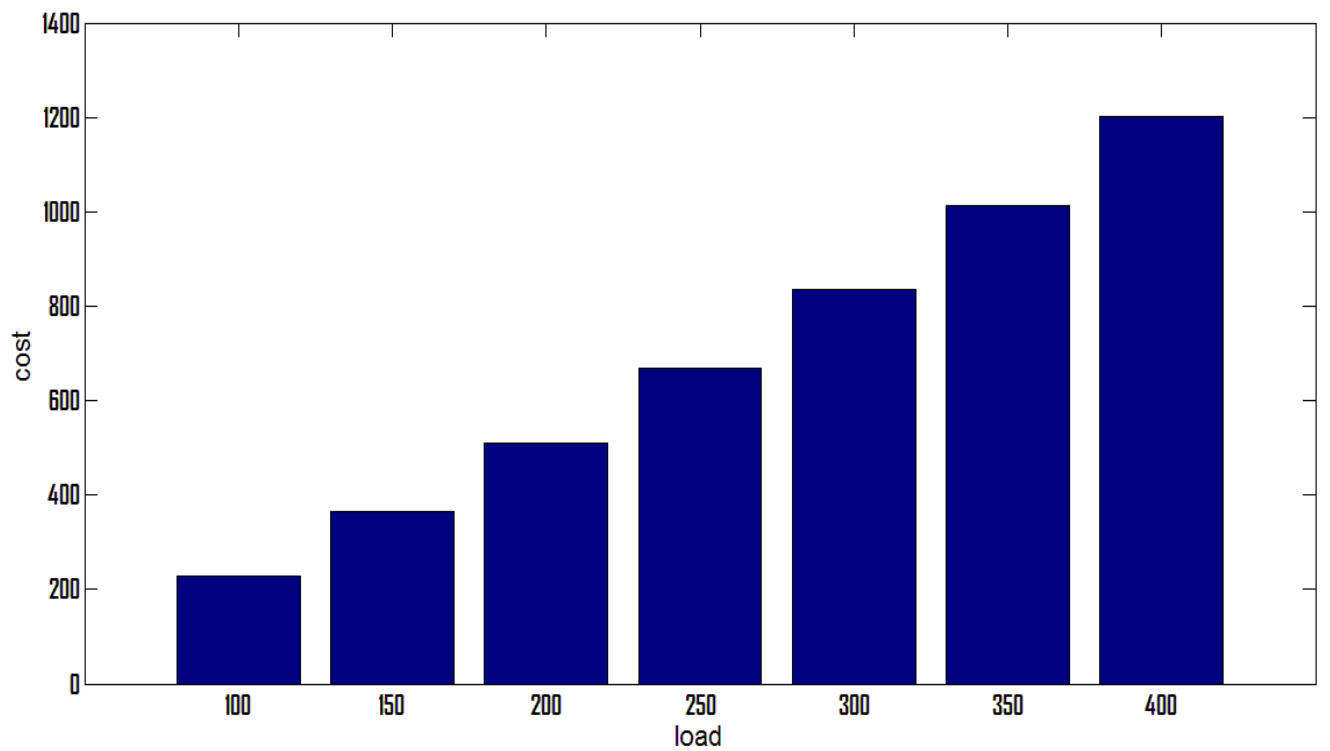

Figure 1. Load Demand v/s total cost

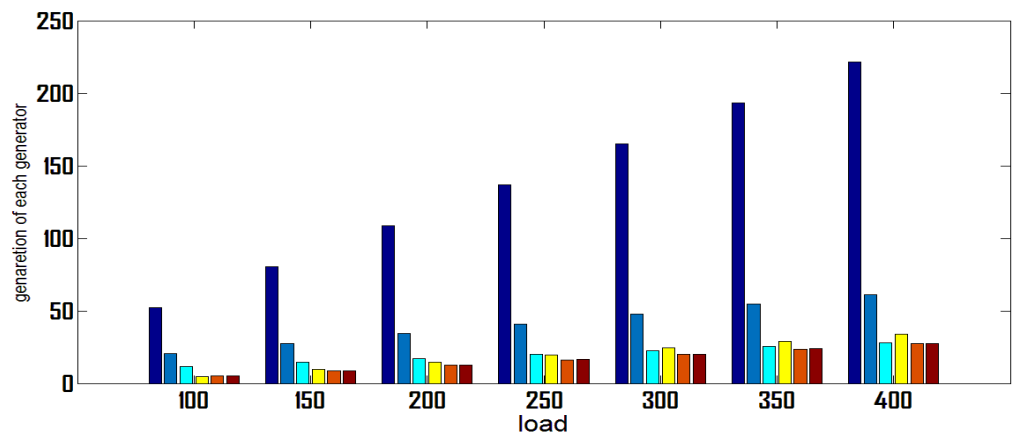

Figure 2. Load Demand v/s generation of each generator 


\subsection{Figures and Tables of Outputs (considering Loss)}

In this section we find out the load sharing of generator s, but here we consider the transmission loss. For this time we have take B-coefficient matrix as an input from the Table 2. Here we also vary our load demand from $100 \mathrm{MW}$ to $400 \mathrm{MW}$ with an interval of $50 \mathrm{MW}$ and get the generation values of each generator for the different load demands. These results are shown in the Table 4.

Table 4.Generator outputs, loss \& costs in different loads

\begin{tabular}{|c|c|c|c|c|c|c|c|c|}
\hline $\begin{array}{c}\mathbf{L o a d} \\
(\mathbf{M W})\end{array}$ & $\begin{array}{c}\boldsymbol{P g}_{1} \\
(\mathbf{M W})\end{array}$ & $\begin{array}{c}\boldsymbol{P g}_{2} \\
(\mathbf{M W})\end{array}$ & $\begin{array}{c}\boldsymbol{P g}_{3} \\
(\boldsymbol{M W})\end{array}$ & $\begin{array}{c}\boldsymbol{P g}_{4} \\
(\mathbf{M W})\end{array}$ & $\begin{array}{c}\boldsymbol{P g}_{5} \\
(\boldsymbol{M W})\end{array}$ & $\begin{array}{c}\boldsymbol{P g}_{\mathbf{6}} \\
(\boldsymbol{M W})\end{array}$ & $\begin{array}{c}\text { Loss } \\
(\boldsymbol{M W})\end{array}$ & $\begin{array}{c}\text { Cost } \\
(\mathbf{R s} / \boldsymbol{h})\end{array}$ \\
\hline 100 & 16.7575 & 16.5460 & 16.7640 & 17.1010 & 16.8088 & 17.1724 & 1.1497 & 277 \\
\hline 150 & 25.2728 & 24.8663 & 25.3083 & 25.8276 & 25.1521 & 26.1789 & 2.6060 & 449.1 \\
\hline 200 & 33.8820 & 33.2199 & 33.9642 & 34.6749 & 33.4550 & 35.4720 & 4.6680 & 644.5 \\
\hline 250 & 42.5875 & 41.6082 & 42.7340 & 43.6456 & 41.7182 & 45.0567 & 7.3503 & 864.3 \\
\hline 300 & 51.3917 & 50.0329 & 51.6206 & 52.7428 & 49.9422 & 54.9382 & 10.6685 & 1109.3 \\
\hline 350 & 60.2974 & 58.4957 & 60.6268 & 61.9694 & 58.1276 & 65.1222 & 14.6392 & 1380.5 \\
\hline 400 & 69.3072 & 66.9985 & 69.7559 & 71.3287 & 66.2752 & 75.6144 & 19.2801 & 1679 \\
\hline
\end{tabular}

The variation of total generation costs for different load demands is shown in the Figure 3 and Figure 4 shows the load shared by the generators at different load demands. The variation of transmission loss at different load demands is also shown graphically in the Figure 5.

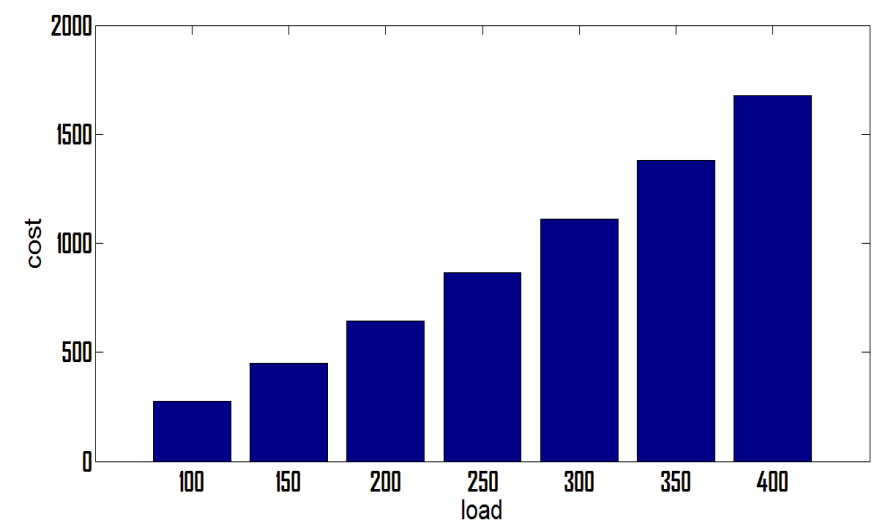

Figure 3. Load Demand v/s total cost

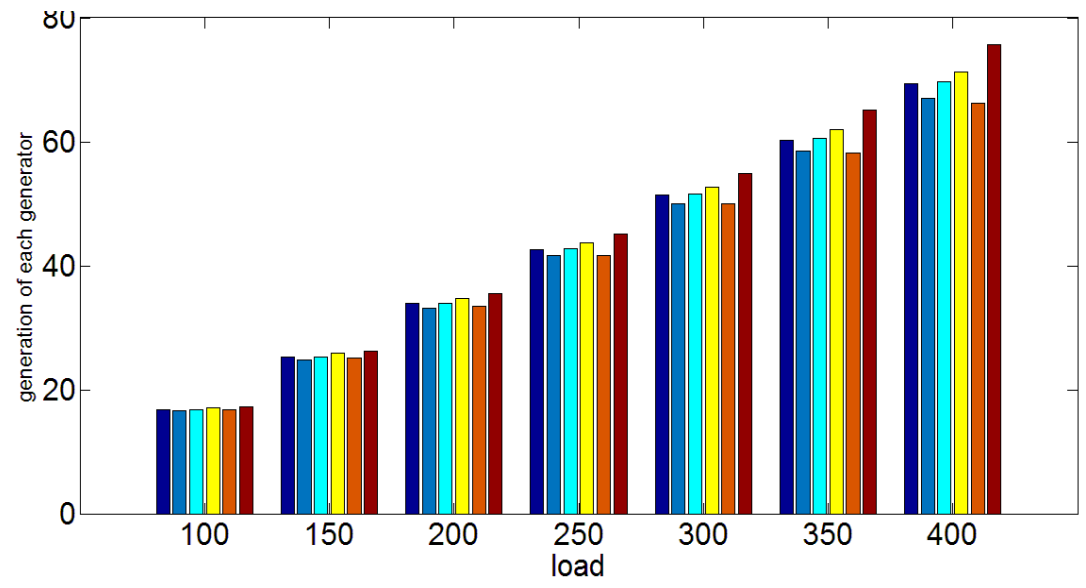

Figure 4. Load Demand v/s generation of each generator 


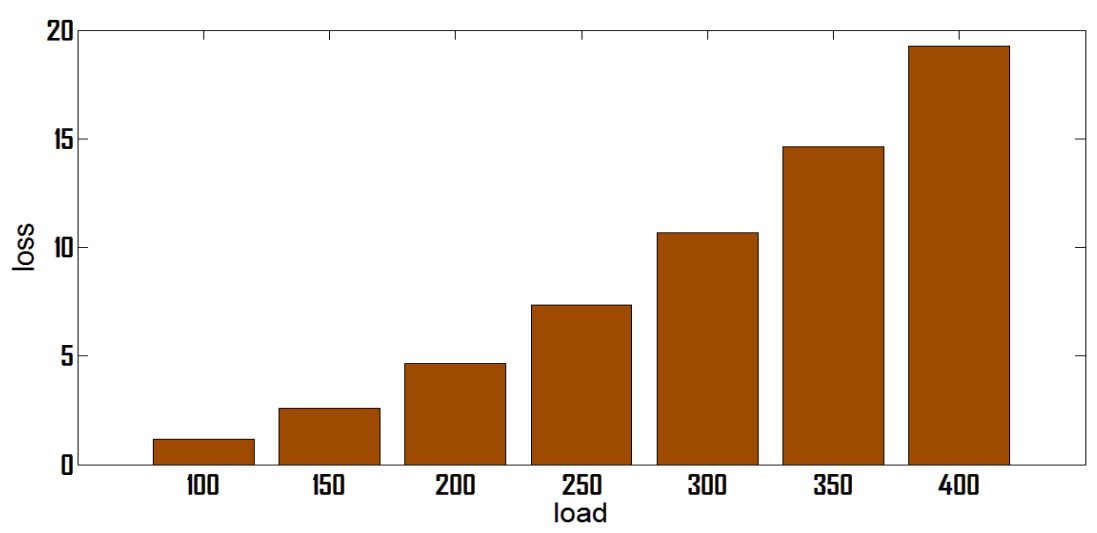

Figure 5. Load Demand v/s Loss

\section{Conclusions}

The demand of power in the world increases day by day. Due to this increase in power demand the emission rate of pollutant increases also. But we have to control this pollution problem and also meet the load demand economically. From this discussion we can conclude that, the balance between economy and emission can be achieved by the Combined Economic and Emission Dispatch, very efficiently.

\section{REFERENCES}

[1] M.E. El-Hawary and G.S. Christensen. "Optimal Economic Operation Of Electric Power Systems"

[2] Sandeep Kaur, G.S. Kochar, D.S. Mahal and Sunita Goyal. "Economic Load Dispatch Solution Using Dynamic Programming",

[3] Jerom. K. Delson and S. M. Shahidehpour "Linear Programming Applications To Power System Economics, Planning And Operation". IEEE Transactions on power system, Vol-7, No-3, August 1992.

[4] J. M. Vignolo and R. Zeballos, "Economic Operation Of Power Systems Optimal Pricing Of Energy"

[5] Dr.A.Allirani, K.Thenmalar and S.Yuvasri. "Economic Thermal Power Dispatch With Emission Constraints Using VSFA Algorithm",

[6] Ugur Güvenç. "Combined Economic Emission Dispatch Solution Using Genetic Algorithm on Similarity Crossover", Scientific Research and Essays, Vol. 5(17), pp. 2451-2456, 4 September, 2010

[7] K.Sathish Kumar, V.Tamilselvan, N.Murali, R,Rajaram, N. Shanmuga Sundaram,and T. Jayabarathi, "Economic Load Dispatch with Emission Constraints using Various PSO Algorithms", International Journal of Recent Trends in Engineering, Vol 2, No. 6, November 2009

[8] "Power System Analysis Operation And Control", By Abhijit Chakrobarti and Sunita Halder (Book).

[9] "Power System Optimization", By D.P. Kothari and J.S. Dhillon (Book).

[10] "Matlab Programming", By Y. Kirani Singh and B.B. Choudhury (Book) 


\section{AUTHORS}

Pratyush Das graduated in Electrical and Electronics Engineering from Bengal College of Engineering and Technology, Durgapur and obtained the M.E degree with specialization of Power System from Bengal Engineering and Science University, Shibpur in 2011. He is an Assistant Professor in Department of Electrical Engineering of Global Institute of Management And Technology, Krishnagar, Nadia, West Bengal

RajuPatwary graduated in Electrical Engineering from Haldia Institute of Technology in 2009 and obtained the M.E degree with specialization of Power System from Bengal Engineering and Science University, Shibpur in 2011. He is an Assistant Professor in Department of Electrical Engineering of Gargi Memorial Institute of Technology Balarampur, Baruipur, 24Parganas (S). He has 2 years teaching experience.

Dr. S.C.Konar graduated in Electrical Engineering from Bengal Engineering College, Shibpur in 1974and obtained the M.E degree with specialization of Control System from Bengal Engineering College, Shibpur in 1979. He has completed his PhD degree from Bengal Engineering College, Shibpur in 2002. He is a Professor in Department of Electrical Engineering of Bengal Engineering and Science University, Shibpur, Howrah, West Bengal
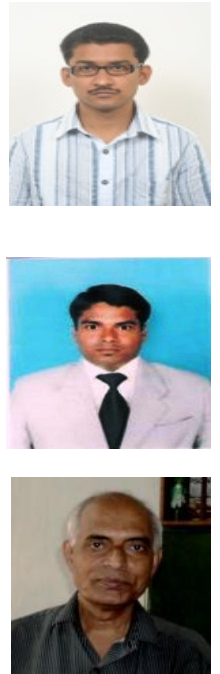